\title{
The Effective Preparation of Secondary Amines Bearing a Vinylsilane Functionality via the Reaction of Primary Amines with $\alpha$-Chlorine-Substituted Allylsilanes Catalyzed by $\mathrm{CuCl}$
}

\author{
Makoto Kozuka and Michiharu Mitani* \\ Department of Chemistry and Material Engineering, Faculty of Engineering, Shinshu University, \\ Wakasato 4-17-1, Nagano 380-8551, Japan
}

The reaction of $\alpha$-chlorine-substituted allylsilanes with primary amines was promoted by $\mathrm{CuCl}$ to result in the selective formation of secondary amines bearing the vinylsilane functionality via the $\mathrm{S}_{\mathrm{N}} 2$ '-allylation holding the silyl group intact.

Key words: vinylsilane, allylsilane, copper catalyst, secondary amine

The preparation of secondary amines has commonly been accomplished by the reactions of primary amines with a range of electrophiles including alkyl halides. Alkylated secondary amines, however, tend to undergo further alkylation because of their enhanced nucleophilicity compared with that of the parent primary amines. Thus, primary amines usually incline to the formation of a complex mixture via multi-alkylation caused by the reaction with electrophiles. Therefore, the selective formation of secondary amines has necessitated some manipulation in order to avoid multi-alkylation, such as the introduction of the protecting group on a nitrogen atom, ${ }^{1}$ the use of primary amines in large excess amounts, ${ }^{2}$ the effecting of reactions via $\pi$-allylpalladium complexes, ${ }^{3}$ and employment of a method utilizing an intermediary generation of a reactive amide anion species. ${ }^{4}$

We have recently reported that the $\mathrm{CuCl}$ catalyst promoted the formation of the three-component coupling products of primary amines with $\alpha$-halogen-substituted allylsilanes and electrophiles such as electron-deficient olefins, alkyl halides, alkyl tosylates or epoxides. ${ }^{5}$ In this investigation, we have obtained an advanced result which disclosed that the selective formation of secondary amines with an allyl group is readily brought about by the $\mathrm{CuCl}$-catalyzed reaction of the primary amines with $\alpha$ -chlorine-substituted allylsilanes in the absence of an other electrophile. A mixture of copper(II) perchlorate and copper metal has been reported to promote the reaction of allyl chlorides with amines to form allyl amines. ${ }^{6}$ In this previous method, however, use of the copper promoter in an equivalent amount was necessitated, and the concomitant formation of diallylated amines in large amounts was brought about. Allylamines are an interesting class of compounds owing to useful synthetic intermediates ${ }^{7}$ along with their physiological properties. ${ }^{8} \quad$ Furthermore, the carbon-carbon double bond of

Correspondence. E-mail: mitanim@shinshu-u.ac.jp 
the allyl group in the obtained secondary amines is substituted with a silyl group to constitute a vinylsilane functionality. Vinylsilanes are building blocks that allow versatile transformations via reactions with a range of electrophiles, ${ }^{9}$ e.g., acyl halides to form conjugated enones.

Initially, (1-chloro-2-methyl-2-propenyl)trimethylsilane 1a was subjected to the reaction with aniline (3 eq.) in a tert- $\mathrm{BuOH}$ solution in the absence of a promoter as a control experiment. The result was a sluggish consumption of $\mathbf{1 a}(700 \mathrm{~min})$ and a formation of the secondary amine 2a, i.e., $N$-(2-methyl-3-trimethylsilyl-2-propenyl)aniline in a low yield (12\%) (Table 1, run 1). Thus, activation of $\mathbf{1 a}$ by a Lewis acid seems to be requisite for the effective allylation of aniline with 1a. However, common Lewis acids may not bring about activation of halides owing to the complex-formation with amines and, furthermore, halogen-containing ones such as $\mathrm{TiCl}_{4}$ are destroyed with protic compounds containing a N-H or O-H bond. Actually, the reaction of $1 \mathbf{a}$ and aniline in the presence of $\mathrm{TiCl}_{4}$ in a tert- $\mathrm{BuOH}$ or $\mathrm{CH}_{2} \mathrm{Cl}_{2}$ solution resulted in no formation of $\mathbf{2 a}$ along with recovery of $\mathbf{1 a}$ (Table 1, runs 2 and 3). The reaction in the presence of $\mathrm{B}(\mathrm{OMe})_{3}$ also failed to derive the enhanced formation of $2 \mathrm{a}$ compared with the reaction without a promoter (Table 1, run 4). Next, copper(I) chloride was examined, resulting in the selective formation of $\mathbf{2 a}(93 \%)$ along with accelerated consumption of 1a. The catalytic activity of the copper(I) halide for the selective formation of 2a proved to descend in order of chloride, bromide and iodide (Table 1, runs 5-7), and copper(II) chloride afforded a low yield of 2a (Table 1, run 8). Furthermore, the reaction using a palladium catalyst, in which the formation of the allyl amine derivatives via a $\pi$-allyl complex might be expected, ${ }^{3}$ was examined. The result after the reaction in 200 min was only recovery of $\mathbf{1 a}$ or the production of $\mathbf{2 a}$ in a trace amount (Table 1, runs 9-11). From the above-mentioned results, it seems certain that copper(I) halide, especially chloride, does not diminish nucleophilicity of the amine via formation of a complex, but rather it enhances the susceptibility of the $\alpha$ -chlorine-substituted allylsilane to nucleophilic attack with the amine.

\section{Scheme 1}

\section{Table 1}

We next investigated the effects of a molar ratio of aniline to 1a on the formation of 2a. While use of an equivalent amount gave an only moderate yield of $\mathbf{2 a}(48 \%)$, use of aniline in two times excess brought about an almost quantitative yield of $\mathbf{2 a}(92 \%)$.

The reactions of some $\alpha$-halogen-substituted allylsilanes with a range of amines were performed under the best conditions of above-mentioned examinations, i.e., use of 2 eq. of an amine and a $\mathrm{CuCl}$ catalyst ( 0.1 eq.) in a tert-BuOH solution. The results are collected in Table 2.

\section{Scheme 2}




\section{Table 2}

(1-Chloro-2-propenyl)trimethylsilane $\mathbf{1 b}$ gave the corresponding secondary amine in an almost quantitative yield by the reaction with aniline in $20 \mathrm{~min}$., similar to the case using 1a (runs 1-2). The reactions of 1a with aniline derivatives bearing alkyl substituents or the amines of aromatic systems other than benzene (i.e., 2-naphtylamine and 2-aminofluorene) also gave the products of type $\mathbf{2}$ in excellent to good yields (runs 3-7). In the reaction of 1a with aniline derivatives bearing an electron-withdrawing group (i.e., nitro or cyano), however, a secondary amine $\mathbf{3}$ based on introduction of a methallyl group without a silyl group was selectively obtained (runs 8 and 9). The basicities of the aniline derivatives bearing those electron-withdrawing groups are low. Thus, hydrogen chloride generated by the reaction with 1a is rather hard to trap with those amines and may promote desilylation of the product of type $\mathbf{2}$ to result in the formation of 3. Also, in the reactions of 1a with $o$-chloaniline or $m$-anisidine, the products of type 3 were formed, although in low yields, along with the products of type $\mathbf{2}$. Formation of $\mathbf{3}$ in these reactions probably results from the lower basicities of $o$-chloroaniline $(\mathrm{pKa}=2.65)$ and $m$-anisidine $(\mathrm{pKa}=$ 4.23) compared with aniline $(\mathrm{pKa}=4.63) .{ }^{10}$ Next, alkyl amines instead of aromatic amines were subjected to the reaction with 1 . As a result, the reactions of $\mathbf{1 a}$ with benzylamine, and $\mathbf{1 b}$ with ethanolamine gave the products of type $\mathbf{2}$ in good yields, although by-production of doubly alkylated tertiary amines 4 in trace amounts was observed (runs 12 and 13). Furthermore, the reaction of the $\alpha$ -bromine-substituted allylsilane 1 $\mathbf{c}$ with ethanolamine brought about diminution in the yield of the product of type 2 and increase of the product of type 4, compared with the reaction of the $\alpha$-chlorine analogue $\mathbf{1 b}$ (runs 13 and 14). While 1c disappeared after 3 hours in the reaction with ethanolamine in the absence of $\mathrm{CuCl}, \mathbf{1 b}$ was not consumed without $\mathrm{CuCl}$ in the reaction time of 3 hours (runs 16 and 17).

Finally, methallyl chloride was subjected to the $\mathrm{CuCl}$-catalyzed reaction with aniline in order to evaluate the effect of the silyl substituent. The result was promotion of multi-allylation compared with the reaction of $1 \mathbf{a}$, as revealed in scheme 3 .

\section{Scheme 3}

The silyl group in $\mathbf{1}$ appears to allow avoidance of multi-alkylation and promote the selective formation of the secondary amine.

\section{Experimemtal}

${ }^{1} \mathrm{H}$ and ${ }^{13} \mathrm{C}$ NMR spectra were recorded on a JNM QX400 (400 and $100 \mathrm{MHz}$ ) spectrometer for solutions in $\mathrm{CDCl}_{3}$ using TMS as internal standard $(\delta=0)$. MS and HRMS were obtained using Shimadzu GCMS-QP5000 and Hitachi M-80B GC-MS instruments equipped with a $30 \mathrm{~m}$ DB-1 columun.

An amine $(2 \mathrm{mmol}), \mathbf{1}(1 \mathrm{mmol}), \mathrm{CuCl}(0.01 \mathrm{~g}, 0.1 \mathrm{mmol})$ and tert-BuOH $(2.5 \mathrm{mmol})$ were added to a 
flask. The resulting solution was stirred under reflux. The reaction mixture was then poured into $\mathrm{H}_{2} \mathrm{O}$ and extracted with $\mathrm{Et}_{2} \mathrm{O}$. After the solvent was mostly removed under reduced pressure, the residue was subjected to column chromatography using hexane/ethyl acetate (1:2) as an eluent.

Compound (2a, E and Z mixture): ${ }^{1} \mathrm{H}$ NMR $\delta \quad 0.05$ (s, 9H, $\mathrm{SiMe}_{3}$ ), 1.74 and 1.82 (s, 3H, MeC=CSi), 3.57 and $3.66\left(\mathrm{~s}, 2 \mathrm{H}, \mathrm{CH}_{2} \mathrm{C}=\mathrm{CSi}\right), 4.78(\mathrm{~s}, 1 \mathrm{H}, \mathrm{NH}), 5.40$ and $5.43(\mathrm{~s}, 1 \mathrm{H}, \mathrm{C}=\mathrm{CHSi}), 6.52-7.10(\mathrm{~m}, 5 \mathrm{H}$, phenyl); ${ }^{13} \mathrm{C}$ NMR $\delta \quad-0.3,19.6,25.2,49.3,53.1,112.5,117.9,128.1,128.7,148.0,152.3$; MS (GC-EI) $\mathrm{m} / \mathrm{z}(\%)$ component $\mathrm{A}=219\left(\mathrm{M}^{+}, 27\right), 204$ (42), 178 (22), 146 (49), 106 (100), 73 (65, 59 (28): component $\mathrm{B}=219$ ( $\left.\mathrm{M}^{+}, 19\right), 204$ (22), 178 (11), 146 (37), 106 (100), 73 (49), 59 (19); (Found: $\mathrm{M}^{+} 219.1473$. $\mathrm{C}_{13} \mathrm{H}_{21} \mathrm{NSi}$ requires M, 219.1443).

Compound (2b): ${ }^{1} \mathrm{H}$ NMR $\delta \quad 0.05$ (s, 9H, $\mathrm{SiMe}_{3}$ ), 3.68 (d, J = 4.8 Hz, 2H, $\mathrm{CH}_{2} \mathrm{CH}=\mathrm{CSi}$ ), 3.90 (s, 1H, $\mathrm{NH}), 5.84(\mathrm{~d}, \mathrm{~J}=18.8 \mathrm{~Hz}, 1 \mathrm{H}, \mathrm{C}=\mathrm{CHSi}), 6.04\left(\mathrm{dt},{ }^{2} \mathrm{~J}=18.8 \mathrm{~Hz},{ }^{3} \mathrm{~J}=4.8 \mathrm{~Hz}, 1 \mathrm{H}, \mathrm{CH}=\mathrm{CSi}\right), 6.49-7.10$ (m, $5 \mathrm{H}$, phenyl); ${ }^{13} \mathrm{C}$ NMR $\delta \quad-0.4,48.5,112.6,117.0,128.6,130.6,142.6,144.9$; MS (GC-EI) m/z (\%) 205 $\left(\mathrm{M}^{+}, 30\right), 190$ (28), 132 (100), 106 (81), 77 (46), 73 (68), 59 (46); (Found: $\mathrm{M}^{+}$205.1283. $\mathrm{C}_{12} \mathrm{H}_{19} \mathrm{NSi}$ requires $\mathrm{M}, 205.1285)$.

Compound (2c, E and Z mixture): ${ }^{1} \mathrm{H}$ NMR $\delta \quad 0.05$ (s, 9H, $\left.\mathrm{SiMe}_{3}\right), 1.05$ (t, J = 7.6 Hz, 3H, $\underline{\mathrm{CH}}_{3} \mathrm{CH}_{2}$ ), 1.68 and 1.77 (s, $3 \mathrm{H}, \mathrm{MeC}=\mathrm{CSi}), 2.40$ (q, J = 7.6 Hz, $\left.\underline{\mathrm{CH}_{2}} \mathrm{CH}_{3}\right), 3.45(\mathrm{~s}, 1 \mathrm{H}, \mathrm{NH}), 3.49$ and 3.59 (s, $2 \mathrm{H}$, $\left.\mathrm{CH}_{2} \mathrm{C}=\mathrm{CSi}\right), 5.34$ and $5.40(\mathrm{~s}, 1 \mathrm{H}, \mathrm{C}=\mathrm{CHSi}), 6.42(\mathrm{~d}, \mathrm{~J}=8.4 \mathrm{~Hz}, 2 \mathrm{H}$, aromaticC $(2) \mathrm{H}), 6.87(\mathrm{~d}, \mathrm{~J}=8.4 \mathrm{~Hz}$, $2 \mathrm{H}$, aromaticC(3)H); ${ }^{13} \mathrm{C}$ NMR $\delta-0.3,15.6,19.3,24.9,27.5,49.3,53.2,112.3,127.5,132.3,145.7$, 152.3; MS (GC-EI) m/z (\%) component A = $247\left(\mathrm{M}^{+}, 38\right), 232$ (56), 206 (35), 174 (35), 134 (1009, 83 (31), 73 (87), 59 (42): component $\mathrm{B}=247\left(\mathrm{M}^{+}, 18\right), 232$ (17), 206 (10), 174 (20), 134 (100), 83 (18), 73 (46), 59 (25); (Found: $\mathrm{M}^{+} 247.1770 . \mathrm{C}_{15} \mathrm{H}_{25} \mathrm{NSi}$ requires 247.1755).

Compound (2d, E and Z mixture ): ${ }^{1} \mathrm{H}$ NMR $\delta \quad 0.05$ (s, 9H, $\mathrm{SiMe}_{3}$ ), 1.70 and 1.79 (s, 3H, MeC=CSi), $1.95(\mathrm{~s}, 3 \mathrm{H}$, aromaticC(4)Me), $2.09(\mathrm{~s}, 3 \mathrm{H}$, aromaticC(2)Me), $3.25(\mathrm{~s}, 1 \mathrm{H}, \mathrm{NH}), 3.55$ and $3.62(\mathrm{~s}, 2 \mathrm{H}$, $\left.\mathrm{CH}_{2} \mathrm{C}=\mathrm{CSi}\right), 5.36$ and $5.40(\mathrm{~s}, 1 \mathrm{H}, \mathrm{C}=\mathrm{CHSi}), 6.40(\mathrm{~d}, \mathrm{~J}=8.4 \mathrm{~Hz}, 1 \mathrm{H}$, aromatic $\mathrm{C}(2) \mathrm{H}), 6.74(\mathrm{~s}, 1 \mathrm{H}$, aromatic $\mathrm{C}(3) \mathrm{H}), 6.79(\mathrm{~d}, \mathrm{~J}=8.4 \mathrm{~Hz}, 1 \mathrm{H}$, aromatic $\mathrm{C}(3) \mathrm{H}) ;{ }^{13} \mathrm{C} \mathrm{NMR} \quad \delta-0.3,17.1,19.3,20.0,25.0,49.3$, 53.1, 109.4, 121.3, 125.5, 126.7, 130.2, 143.5, 152.4; MS (GC-EI) m/z (\%) component A = $247\left(\mathrm{M}^{+}, 36\right)$, 232 (31), 206 (8), 190 (11), 174 (55), 134 (100), 73 (64), 59 (37): component $\mathrm{B}=247$ ( $\left.\mathrm{M}^{+}, 22\right), 232$ (10), 206 (5), 190 (5), 174 (22), 134 (100), 73 (57), 59 (25); (Found: $\mathrm{M}^{+}$247.1759. $\mathrm{C}_{15} \mathrm{H}_{25} \mathrm{NSi}$ requires 247.1755).

Compound (2e, E and Z mixture): ${ }^{1} \mathrm{H}$ NMR $\delta \quad 0.05$ (s, 9H, $\mathrm{SiMe}_{3}$ ), 1.75 and 1.77 (s, 3H, MeC=CSi), $2.11(\mathrm{~s}, 3 \mathrm{H}$, aromatic $\mathrm{C}(4) \mathrm{Me}), 2.19$ (s, $6 \mathrm{H}$, aromatic $\mathrm{C}(2)$ and $\mathrm{C}(6) \mathrm{Me}), 3.31$ and $3.44\left(\mathrm{~s}, 2 \mathrm{H}, \mathrm{CH}_{2} \mathrm{C}=\mathrm{CSi}\right)$, 5.30 and $5.51(\mathrm{~s}, 1 \mathrm{H}, \mathrm{C}=\mathrm{CHSi}), 6.70(\mathrm{~s}, 2 \mathrm{H}$, aromatic $\mathrm{C}(3)$ and $\mathrm{C}(5) \mathrm{H}) ;{ }^{13} \mathrm{C}$ NMR $\delta \quad-0.4,18.1,18.4$, 20.4, 25.4, 53.8, 57.9, 123.1, 127.7, 129.0, 130.7, 143.4, 152.3; MS (GC-EI) m/z (\%) component A = 261 $\left(\mathrm{M}^{+}, 50\right), 246$ (29), 188 (74), 148 (100), 134 (59), 91 (24), 73 (55), 59 (37): component B = $261\left(\mathrm{M}^{+}, 20\right)$, 246 (7), 188 (30), 105 (100), 134 (28), 91 (13), 73 (30), 59 (24); (Found: $\mathrm{M}^{+} 261.1934 . \mathrm{C}_{16} \mathrm{H}_{27} \mathrm{NSi}$ requires 261.1911).

Compound (2f, E and Z mixture): ${ }^{1} \mathrm{H}$ NMR $\delta \quad 0.05$ (s, 9H, SiMe 3 ), 1.70 and 1.81 (s, 3H, MeC=CSi), 
3.61 and $3.71\left(\mathrm{~s}, 2 \mathrm{H}, \mathrm{CH}_{2} \mathrm{C}=\mathrm{CSi}\right), 4.30(\mathrm{~s}, 1 \mathrm{H}, \mathrm{NH}), 5.42$ and $5.44(\mathrm{~s}, 1 \mathrm{H}, \mathrm{C}=\mathrm{CHSi}), 6.45-7.61(\mathrm{~m}, 7 \mathrm{H}$, naphthyl); ${ }^{13} \mathrm{C}$ NMR $\delta \quad-0.3,19.7,25.2,49.2,52.0,104.0,117.0,119.2,122.8,124.1,125.1,126.0,128.0$, 128.1, 133.7, 142.8, 151.8; MS (GC-EI) m/z (\%) component A = $269\left(\mathrm{M}^{+}, 67\right), 254$ (42), 196 (62), 156 (46), 127 (25), 92 (40), 73 (100), 59 (49): component $\mathrm{B}=269\left(\mathrm{M}^{+}, 40\right), 254$ (21), 196 (48), 156 (65), 127 (33), 115 (26), 73 (100), 59 (52); (Found: $\mathrm{M}^{+}$269.1578. $\mathrm{C}_{17} \mathrm{H}_{23} \mathrm{NSi}$ requires 269.1597).

Compound (2g, E and Z mixture): ${ }^{1} \mathrm{H}$ NMR $\delta \quad 0.05$ (s, 9H, SiMe $), 1.72$ and 1.78 (s, 3H, MeC=CSi), 3.51 and $3.60\left(\mathrm{~s}, 2 \mathrm{H}, \mathrm{CH}_{2} \mathrm{C}=\mathrm{CSi}\right), 3.62\left(\mathrm{~s}, 2 \mathrm{H}\right.$, fluoren $\left.\mathrm{CH}_{2}\right), 3.87(\mathrm{~s}, 1 \mathrm{H}, \mathrm{NH}), 5.34$ and $5.37(\mathrm{~s}, 1 \mathrm{H}$, $\mathrm{C}=\mathrm{CHSi}), 6.43-7.44\left(\mathrm{~m}, 7 \mathrm{H}\right.$, benzene ring); ${ }^{13} \mathrm{C} \mathrm{NMR} \quad \delta-0.3,20.1,24.9,36.5,49.3,49.7,108.6,111.4$, $117.7,119.9,124.0,125.9,127.7,131.3,141.4,141.6,144.4,147.3,152.0 ;$ MS (GC-EI) m/z (\%) component $\mathrm{A}=307\left(\mathrm{M}^{+}, 94\right), 292$ (59), 266 (51), 234 (33), 194 (64), 180 (36), 165 (47), 73 (100): component B = $307\left(\mathrm{M}^{+}, 74\right), 292$ (25), 266 (18), 234 (25), 194 (100), 180 (29), 165 (37), 73 (51); (Found: $\mathrm{M}^{+}$307.1785. $\mathrm{C}_{20} \mathrm{H}_{25} \mathrm{NSi}$ requires 307.1755).

Compound (3h): ${ }^{1} \mathrm{H}$ NMR $\delta 1.71$ (s, 3H, $\left.\mathrm{MeC}=\mathrm{C}\right), 2.95$ (s, $\left.1 \mathrm{H}, \mathrm{NH}\right), 3.71$ (s, $\left.2 \mathrm{H}, \mathrm{CH}_{2} \mathrm{C}=\mathrm{C}\right), 4.86$ (s, $\left.2 \mathrm{H}, \mathrm{C}=\mathrm{CH}_{2}\right), 6.46(\mathrm{~d}, \mathrm{~J}=8.4 \mathrm{~Hz}, 2 \mathrm{H}$, aromatic $\mathrm{C}(2)$ and $\mathrm{C}(6) \mathrm{H}), 7.99(\mathrm{~d}, \mathrm{~J}=8.4 \mathrm{hz}$, aromatic $\mathrm{C}(3)$ and $\mathrm{C}(5) \mathrm{H}) ;{ }^{13} \mathrm{C}$ NMR $\delta \quad 20.3,49.2,111.1,111.6,126.2,137.6,140.6,153.0 ; \mathrm{MS}(\mathrm{DI}) \mathrm{m} / \mathrm{z}(\%) 192\left(\mathrm{M}^{+}, 45\right)$, 177 (25), 151 (100), 130 (14), 105 (45), 76 (15), 55 (30); (Found: $\mathrm{M}^{+}$192.0917. $\mathrm{C}_{10} \mathrm{H}_{12} \mathrm{~N}_{2} \mathrm{O}_{2}$ requires 192.0898).

Compound (3i): ${ }^{1} \mathrm{H}$ NMR $\delta 1.63(\mathrm{~s}, 3 \mathrm{H}, \mathrm{MeC}=\mathrm{C}), 3.58\left(\mathrm{~s}, 2 \mathrm{H}, \mathrm{CH}_{2} \mathrm{C}=\mathrm{C}\right), 4.42(\mathrm{~s}, 1 \mathrm{H}, \mathrm{NH}), 4.78(\mathrm{~s}, 2 \mathrm{H}$, $\left.\mathrm{C}=\mathrm{CH}_{2}\right), 6.42(\mathrm{~d}, \mathrm{~J}=8.9 \mathrm{~Hz}, 2 \mathrm{H}$, aromatic $\mathrm{C}(2)$ and $\mathrm{C}(6) \mathrm{H}), 7.26(\mathrm{~d}, \mathrm{~J}=8.9 \mathrm{~Hz}$, aromatic $\mathrm{C}(3)$ and $\mathrm{C}(5) \mathrm{H}) ;{ }^{13} \mathrm{C}$ NMR $\delta \quad 20.1,49.0,109.5,111.6,122.3,114.5,133.7,133.9,141.3$; MS (GS-EI) m/z (\%) $172\left(\mathrm{M}^{+}, 62\right), 157$ (71), 131 (100), 102 (19), 55 (23); (Found: $\mathrm{M}^{+}$172.0971. $\mathrm{C}_{11} \mathrm{H}_{12} \mathrm{~N}_{2}$ requires 172.0999).

Compound (2j, E and Z mixture): ${ }^{1} \mathrm{H}$ NMR $\delta 0.06$ (s, 9H, SiMe 3 ), 1.68 and 1.79 (s, 3H, MeC=CSi), 3.59 and $3.67\left(\mathrm{~s}, 2 \mathrm{H}, \mathrm{CH}_{2} \mathrm{C}=\mathrm{CSi}\right), 4.23(\mathrm{~s}, 1 \mathrm{H}, \mathrm{NH}), 5.41$ and $5.44(\mathrm{~s}, 1 \mathrm{H}, \mathrm{C}=\mathrm{CHSi}), 6.46-7.13(\mathrm{~m}, 4 \mathrm{H}$, aromatic); ${ }^{13} \mathrm{C}$ NMR $\delta-0.86,20.0,24.9,49.1,52.4,110.5,111.0,116.6,118.4,127.1,128.4,143.6$, 151.3; MS (GC-EI) m/z (\%) component $\mathrm{A}=255\left[\mathrm{M}\left(\mathrm{Cl}^{37}\right)^{+}, 8\right], 253\left[\mathrm{M}\left(\mathrm{Cl}^{35}\right)^{+}, 21\right], 238$ (46), $180(44), 140$ (53), 73 (100), 59 (38): component $\mathrm{B}=255\left[\mathrm{M}\left(\mathrm{Cl}^{37}\right)^{+}, 10\right], 253\left[\mathrm{M}\left(\mathrm{Cl}^{35}\right)^{+}, 28\right], 238$ (33), 180 (53), 140 (76), 73 (100), 59 (30); [Found: $\mathrm{M}\left(\mathrm{Cl}^{35}\right)^{+} 253.1039 . \mathrm{C}_{13} \mathrm{H}_{20} \mathrm{NSiCl}$ requires 253.1052].

Compound (2k, E and Z mixture): ${ }^{1} \mathrm{H}$ NMR $\delta \quad 0.05$ (s, 9H, SiMe $), 1.68$ and 1.77 (s, 3H, MeC=CSi), $3.51(\mathrm{~s}, 1 \mathrm{H}, \mathrm{NH}), 3.58$ and $3.61\left(\mathrm{~s}, 2 \mathrm{H}, \mathrm{CH}_{2} \mathrm{C}=\mathrm{CSi}\right), 3.61(\mathrm{~s}, 3 \mathrm{H}, \mathrm{OMe}), 5.34$ and $5.39(\mathrm{~s}, 1 \mathrm{H}, \mathrm{C}=\mathrm{CHSi})$, $6.04(\mathrm{~s}, 1 \mathrm{H}$, aromatic $\mathrm{C}(2) \mathrm{H}), 6.09-6.95(\mathrm{~m}, 3 \mathrm{H}$, aromatic $\mathrm{C}(4), \mathrm{C}(5)$ and $\mathrm{C}(6) \mathrm{H}) ;{ }^{13} \mathrm{C}$ NMR $\delta-1.0,19.5$, 24.9, 49.0, 52.9, 54.5, 98.2, 102.0, 105.4, 127.8, 129.3, 149.1, 152.0, 160.0; MS (GC-EI) m/z (\%) component $\mathrm{A}=249\left(\mathrm{M}^{+}, 40\right), 234(50), 176(68), 136$ (100), 73 (85): component $\mathrm{B}=249\left(\mathrm{M}^{+}, 30\right), 234$ (25), 176 (42), 136 (100), 73 (48); (Found: $\mathrm{M}^{+} 249.1559 . \mathrm{C}_{14} \mathrm{H}_{23} \mathrm{NOSi}$ requires 249.1548).

Compound (2I, E and Z mixture): ${ }^{1} \mathrm{H}$ NMR $\delta 0.07$ (s, 9H, SiMe 3 ), 1.67 and 1.79 (s, 3H, MeC=CSi), 3.06 and $3.14\left(\mathrm{~s}, 2 \mathrm{H}, \mathrm{CH}_{2} \mathrm{C}=\mathrm{CSi}\right), 3.61\left(\mathrm{~s}, 2 \mathrm{H}, \mathrm{PhCH}_{2} \mathrm{~N}\right), 3.98(\mathrm{~s}, 1 \mathrm{H}, \mathrm{NH}), 5.25$ and $5.32(\mathrm{~s}, 1 \mathrm{H}, \mathrm{C}=\mathrm{CHSi})$, 7.18 (s, 5H, phenyl); ${ }^{13} \mathrm{C}$ NMR $\delta \quad-1.0,19.9,25.0,53.0,54.2,58.2,126.4,127.3,127.7,128.0,140.1$, 153.2; MS (GC-EI) m/z (\%) component A = 218 (6), 178 (10), 160 (14), 142 (35), 120 (24), 91 (100), 73 
(68): component B = 218 (5), 178 (10), 160 (15), 142 (25), 120 (32), 91 (100), 73 (37); MS (GC-CI) m/z component $\mathrm{A}=234(\mathrm{M}+1)^{+}$: component $\mathrm{B}=234(\mathrm{M}+1)^{+} ;$[Found: $(\mathrm{M}-\mathrm{Me})^{+} 218.1369 . \mathrm{C}_{13} \mathrm{H}_{20} \mathrm{NSi}$ requires 218.1364$]$.

Compound (2m): ${ }^{1} \mathrm{H}$ NMR $\delta \quad 0.05$ (s, 9H, $\mathrm{SiMe}_{3}$ ), 2.61 (t, J = 5.6 Hz, 2H, $\left.\underline{\mathrm{NCH}}_{2} \mathrm{CH}_{2} \mathrm{OH}\right), 2.80(\mathrm{~s}, 2 \mathrm{H}$, $\mathrm{OH}$ and $\mathrm{NH}), 3.08$ (d, J = 4.8 Hz, 2H, $\left.\underline{\mathrm{NCH}}_{2} \mathrm{CH}=\mathrm{C}\right), 3.53\left(2 \mathrm{H}, \mathrm{J}=5.6 \mathrm{~Hz}, 2 \mathrm{H}, \mathrm{NCH}_{2} \underline{\mathrm{CH}}_{2} \mathrm{OH}, 5.62(\mathrm{~d}, \mathrm{~J}=\right.$ $18.8 \mathrm{~Hz}, 1 \mathrm{H}, \mathrm{C}=\mathrm{CHSi}), 5.83\left(\mathrm{dt},{ }^{2} \mathrm{~J}=18.8 \mathrm{~Hz},{ }^{3} \mathrm{~J}=4.8 \mathrm{~Hz}, 1 \mathrm{H}, \mathrm{CH}=\mathrm{CSi}\right) ;{ }^{13} \mathrm{C}$ NMR $\delta-1.0,50.5,54.2$, 60.6, 123.1, 127.9; MS (GC-EI) m/z (\%) $173\left(\mathrm{M}^{+}, 8\right), 158$ (10), 142 (36), 128 (17), 73 (100), 59 (42); (Found: $\mathrm{M}^{+}$173.3021. $\mathrm{C}_{8} \mathrm{H}_{19} \mathrm{NOSi}$ requires 173.3042).

\section{References}

1 a) J. Jacobus, M. Raban and K. Mislow, J. Org. Chem., 1968, 33, 1142. b) P. Nussbaumer, K. Baumann, T. Dechat and M. Harasek, Tetrahedron, 1991, 47, 4591. c) M. B. Sassaman, Tetrahedron, 1996, 52, 10835.

2 a) J. H. Speer and A. J. Hill, J. Org. Chem., 1937, 2, 139. b) W. J. Hickinbottom, J. Chem. Soc., 1930, 992.

3 a) S.-I. Murahashi, Y. Imada, Y. Taniguchi and Y. Kodera, Terahedron Lett., 1988, 29, 2973 . b) S.-C. Yang and C.-W. Hung, Synthesis, 1999, 1747.

4 W. Ando and H. Tsumaki, Chem. Lett., 1981, 693.

5 M. Kozuka, A. Inoue, T. Tsuchida and M. Mitani, Adv. Synth. Cat., 2006, 348, 953.

6 J. B. Baruah and A. G. Samuelson, Tetrahedron, 1991, 47, 9449.

7 A. Stutz, Angew. Chem., Int. Ed. Engl., 1987, 26, 320.

8 a) Y. Genisson, M. Mehmandoust, C. Marazano and B. C. Das, Heterocycles, 1994, 39, 811. b) P. Jain, H. M. Garraffo, T. F. Spande, H. J. C. Yeh and J. W. Daly, J. Nat. Prod., 1995, 58, 100.

9 a) E. W. Colvin, Silicon Reagents in Organic Synthesis, Academic Press, London, 1988, p. 14. b) I. Fleming, A. Barbero and D. Walter, Chem. Rev., 1997, 97, 2063.

10 CRC Handbook of Chemistry and Physics, ed by D. R. Lide, CRC Press, Boca Raton, 1993, 8-43. 
<smiles>C=C(C)C(Cl)[As]</smiles>

$1 \mathbf{a}$

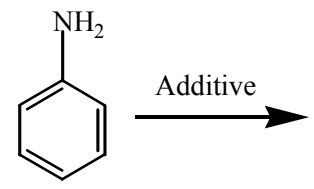<smiles>CC(C)=CC(C)=CNc1ccccc1</smiles>

Scheme 1

Table 1. Effect of metallic species on the reaction of 1a and aniline ${ }^{a}$

\begin{tabular}{|c|c|c|c|c|}
\hline Entry & Additive & solvent & Time $/ \min ^{\mathrm{b}}$ & $\mathbf{2} \mathbf{a}^{\mathrm{c}}$ Yield $/ \%{ }^{\mathrm{C}}$ \\
\hline 1 & none & t-BuOH & 700 & 12 \\
\hline 2 & $\mathrm{TiCl}_{4}$ & t-BuOH & 100 & 0 \\
\hline 3 & $\mathrm{TiCl}_{4}$ & $\mathrm{CH}_{2} \mathrm{Cl}_{2}$ & 100 & 0 \\
\hline 4 & $\mathrm{~B}(\mathrm{OMe})_{3}$ & $\mathrm{t}-\mathrm{BuOH}$ & 700 & 12 \\
\hline 5 & $\mathrm{CuCl}$ & $\mathrm{t}-\mathrm{BuOH}$ & 20 & $93(92)^{e}$ \\
\hline 6 & $\mathrm{CuBr}$ & r-BuOH & 20 & 65 \\
\hline 7 & $\mathrm{CuI}$ & t-BuOH & 20 & 20 \\
\hline 8 & $\mathrm{CuCl}_{2}$ & t-BuOH & 20 & 25 \\
\hline 9 & $\mathrm{Pd}(\mathrm{OAc})_{2}{ }^{\mathrm{f}}$ & DMF & 200 & 7 \\
\hline 10 & $\mathrm{Pd}(\mathrm{OAc})_{2}{ }^{\mathrm{f}}$ & $\mathrm{t}-\mathrm{BuOH}$ & 200 & 7 \\
\hline 11 & $\mathrm{Pd}(\mathrm{OAc})_{2}{ }^{\mathrm{f}}$ & $\mathrm{CH}_{3} \mathrm{CN}$ & 200 & 0 \\
\hline \multicolumn{5}{|c|}{$\begin{array}{l}{ }^{\mathrm{a}} \text { Reaction conditions; 1a }(1 \mathrm{mmol}) \text {, aniline (3 mmol), } \\
\text { additive }(0.2 \mathrm{mmol}) \text {, solvent }(2.5 \mathrm{ml}) \text {. }{ }^{\mathrm{b}} \text { Time at which 1a } \\
\text { was consumed. }{ }^{\mathrm{c}} \mathrm{A} 3 / 1 \text { mixture of }(\mathrm{E}) \text { and }(\mathrm{Z}) \\
\text { stereoisomers. } \\
{ }^{\mathrm{e}} \text { Determioned by GC analysis. } \\
\text { the presence of } \mathrm{PPh}_{3} \text { as a ligand. }\end{array}$} \\
\hline
\end{tabular}




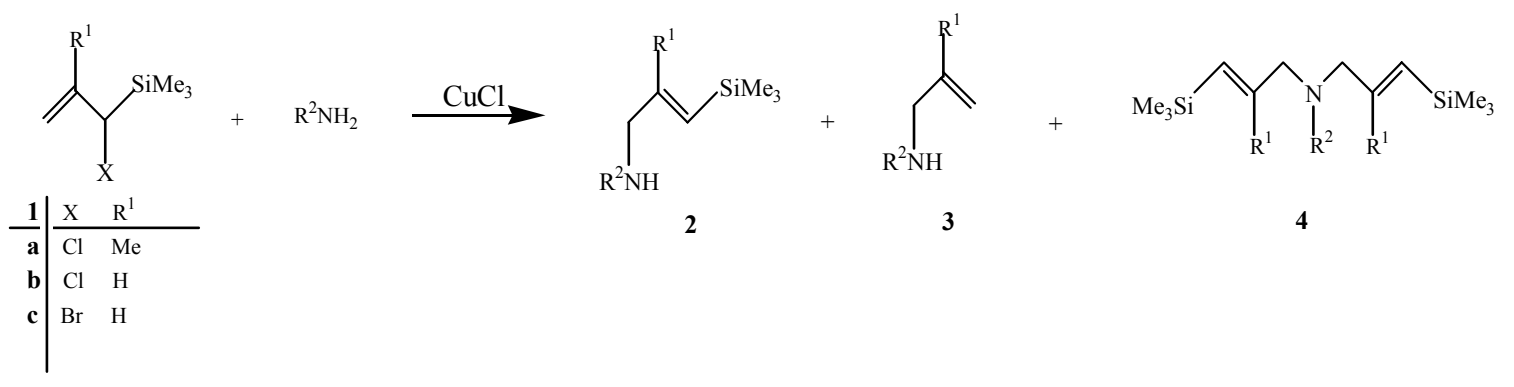

Scheme 2

Table 2. Reaction of $\alpha$-chlorine substituted allylsilanes with amines ${ }^{\mathrm{a}}$

\begin{tabular}{|c|c|c|c|c|c|c|c|}
\hline \multirow[t]{2}{*}{ Entry } & \multirow[t]{2}{*}{1} & \multirow[t]{2}{*}{$\mathrm{R}^{2} \mathrm{NH}_{2}$} & \multirow[t]{2}{*}{ Time $/ \mathrm{min}^{\mathrm{b}}$} & \multicolumn{4}{|c|}{ Yield $/ \%{ }^{\mathrm{c}}$} \\
\hline & & & & & & 3 & 4 \\
\hline 1 & $\mathbf{a}$ & $\mathrm{PhNH}_{2}$ & 20 & $\mathbf{a}^{\mathrm{d}}$ & 92 & 0 & 0 \\
\hline 2 & $\mathbf{b}$ & $\mathrm{PhNH}_{2}$ & 20 & $\mathbf{b}$ & 90 & 0 & 0 \\
\hline 3 & $\mathbf{a}$ & $4-\mathrm{EtC}_{6} \mathrm{H}_{4} \mathrm{NH}_{2}$ & 25 & $\mathbf{c}^{\mathrm{d}}$ & 90 & 0 & 0 \\
\hline 4 & $\mathbf{a}$ & $2,4-\mathrm{Me}_{2} \mathrm{C}_{6} \mathrm{H}_{3} \mathrm{NH}_{2}$ & 25 & $d^{\mathrm{e}}$ & 95 & 0 & 0 \\
\hline 5 & $\mathbf{a}$ & $2,4,6-\mathrm{Me}_{3} \mathrm{C}_{6} \mathrm{H}_{2} \mathrm{NH}_{2}$ & 35 & $e^{f}$ & 90 & 0 & 0 \\
\hline 6 & $\mathbf{a}$ & 2-naphthylamine & 45 & $f^{d}$ & 94 & 0 & 0 \\
\hline $7^{g}$ & $\mathbf{a}$ & 2-aminofluorene & 20 & $\mathrm{~g}^{\mathrm{h}}$ & 90 & 0 & 0 \\
\hline 8 & $\mathbf{a}$ & $4-\mathrm{NO}_{2} \mathrm{C}_{6} \mathrm{H}_{4} \mathrm{NH}_{2}$ & 35 & h & 0 & 88 & 0 \\
\hline $9^{i}$ & $\mathbf{a}$ & $4-\mathrm{NCC}_{6} \mathrm{H}_{4} \mathrm{NH}_{2}$ & 40 & i & 0 & 85 & 0 \\
\hline $10^{i}$ & $\mathbf{a}$ & $2-\mathrm{ClC}_{6} \mathrm{H}_{4} \mathrm{NH}_{2}$ & 15 & $\mathbf{j}^{\mathrm{d}}$ & 62 & 31 & 0 \\
\hline 11 & $\mathbf{a}$ & $3-\mathrm{MeOC}_{6} \mathrm{H}_{4} \mathrm{NH}_{2}$ & 25 & $\mathbf{k}^{j}$ & 72 & 25 & 0 \\
\hline 12 & $\mathbf{a}$ & $\mathrm{PhCH}_{2} \mathrm{NH}_{2}$ & 40 & $\mathbf{l}^{\mathrm{f}}$ & 83 & 0 & 1.5 \\
\hline 13 & $\mathbf{b}$ & $\mathrm{HO}\left(\mathrm{CH}_{2}\right)_{2} \mathrm{NH}_{2}$ & 60 & $\mathbf{m}$ & 88 & 0 & 1 \\
\hline 14 & c & $\mathrm{HO}\left(\mathrm{CH}_{2}\right)_{2} \mathrm{NH}_{2}$ & 40 & $\mathbf{m}$ & 61 & 0 & 10 \\
\hline $15^{\mathrm{k}}$ & c & $\mathrm{HO}\left(\mathrm{CH}_{2}\right)_{2} \mathrm{NH}_{2}$ & 180 & $\mathbf{m}$ & 65 & 0 & 13 \\
\hline $16^{\mathrm{K}}$ & b & $\mathrm{HO}\left(\mathrm{CH}_{2}\right)_{2} \mathrm{NH}_{2}$ & $180^{1}$ & $\mathbf{m}$ & 0 & 0 & 0 \\
\hline
\end{tabular}

${ }^{\mathrm{a}}$ Reaction conditions; $1 \mathrm{a}(1 \mathrm{mmol})$, amine $(2 \mathrm{mmol}), \mathrm{CuCl}(0.1 \mathrm{mmol}), \mathrm{t}-\mathrm{BuOH}(2.5 \mathrm{ml})$ under reflux. ${ }^{\mathrm{b}}$ Time at which 1 was consumed. ${ }^{\mathrm{c}}$ Determined by column chromatography isolation. ${ }^{\mathrm{d}} \mathrm{E} / \mathrm{Z}=3 / 1$. ${ }^{\mathrm{e}} \mathrm{E} / \mathrm{Z}=$ 2/1. ${ }^{\mathrm{f}} \mathrm{E} / \mathrm{Z}=1 / 1 .{ }^{\mathrm{g}}$ Conditions; $1 \mathrm{a}(1 \mathrm{mmol}) / \mathrm{amine}(2 \mathrm{mmol}) / \mathrm{CuCl}(0.1 \mathrm{mmol}) / \mathrm{EtOH}(12 \mathrm{ml}) .{ }^{\mathrm{h}} \mathrm{E} / \mathrm{Z}=5 / 1$. ${ }^{\mathrm{i}}$ Conditions; $1 \mathrm{a}(0.5 \mathrm{mmol}) /$ amine $(2 \mathrm{mmol}) / \mathrm{CuCl}(0.1 \mathrm{mmol}) / \mathrm{EtOH}(2.5 \mathrm{ml}) .{ }^{\mathrm{j}} \mathrm{E} / \mathrm{Z}=4 / 1 .{ }^{\mathrm{k}} \mathrm{Without} \mathrm{CuCl}$. 1b was not consumed.<smiles>C=C(C)CCl</smiles>

Scheme 3 